\title{
Efectos de la fertilización del suelo sobre el crecimiento arbóreo en bosques pluviales tropicales del Chocó, Colombia
}

\author{
Harley Quinto Mosquera ${ }^{1}$, Yeison Rivas Urrutia ${ }^{1} \&$ Flavio Moreno Hurtado ${ }^{2}$ \\ 1. Programa de Biología. Facultad de Ciencias Naturales, Universidad Tecnológica del Chocó "Diego Luis Córdoba", \\ 22 №18B-10, Quibdó, Colombia; hquintom@gmail.com, yeisonrivas29@gmail.com \\ 2. Departamento de Ciencias Forestales, Facultad de Ciencias Agrarias, Universidad Nacional de Colombia Sede \\ Medellín, Medellín, Colombia; fhmoreno@unal.edu.co
}

Recibido 02-XI-2016. Corregido 12-IV-2017. Aceptado 12-V-2017.

\begin{abstract}
Effects of soil fertilization on tree growth in tropical rainforests of Choco, Colombia. The tree diameter growth (CDA) is a fundamental component of net primary productivity (NPP) and carbon storage in forest ecosystems; therefore, it plays a key role in mitigating global climate change. It has been hypothesized that CDA in lowland tropical rain forests is limited by the availability of soil nutrients, yet little experimental evidence is available, especially in forest of high precipitation $(>5000 \mathrm{~mm}$ per year). This study evaluated the effects of soil fertilization on CDA in tropical rainforests of the Colombian Pacific, one of the wettest areas of the world. Such effects were assessed at the level of localities, diametric categories, wood density, and functional groups. To do this, two arboreal inventories (2014 and 2015) were performed, five fertilization treatments (Nitrogen-N, Phosphorus-P, Potassium-K, NPK and Control) were applied, and the CDA was determined in five permanent plots of one hectare. We found that the application of N and NPK had little effect on CDA as compared to the control; while the addition of $\mathrm{P}$ and $\mathrm{K}$ produced significant reduction of the trees relative growth rate, with respect to the control at the level of localities, in small and medium sized trees, in all wood densities (low, medium and high), and in the group of dicotyledonous species. Although these results did not corroborate the hypothesis of nutrient limitation on CDA in the studied forests, it was found that during the early years of fertilization (mainly with P and K), different patterns of aboveground and belowground NPP occurred to maximize photosynthesis and soil nutrient acquisition. Rev. Biol. Trop. 65 (3): 1161-1173. Epub 2017 September 01.
\end{abstract}

Key words: carbon balance, biogeographic Chocó, nitrogen, phosphorus, potassium, relative growth rate, net primary productivity.

Los bosques tropicales, debido a su alta productividad primaria neta $(\mathrm{PPN})$ y a su papel como sumideros y reservorios de carbono atmosférico, tienen una función significativa en el balance del carbono global y en la mitigación del cambio climático (Dixon et al., 1994; Phillips et al., 1998; IPCC, 2014). Sin embargo, la capacidad que tienen estos bosques de capturar $\mathrm{CO}_{2}$ atmosférico está determinada por diversos factores biológicos y ambientales, que aún no están plenamente comprendidos (Schuur, 2003; Cleveland et al., 2011). Particularmente, la disponibilidad de nutrientes del suelo, como nitrógeno $(\mathrm{N})$, fósforo $(\mathrm{P})$ y potasio $(\mathrm{K})$, limita la PPN de estos ecosistemas (Vitousek \& Farrington, 1997; Paoli \& Curran, 2007; Cleveland et al., 2011; Wright et al., 2011). Sin embargo, dicha limitación nutricional aún se encuentra en evaluación y análisis, pues las evidencias directas son muy pocas y muestran incertidumbres y posibles contradicciones (Sullivan et al., 2014; Dalling, Heineman, López, Wright, \& Turner, 2016).

En tal sentido, se ha planteado la hipótesis de que en bosques lluviosos tropicales de baja altitud la PPN es limitada principalmente por la disponibilidad de $\mathrm{P}$ edáfico, debido a las altas tasas de meteorización del material 
parental, que facilitan las pérdidas del mineral por lixiviación, y además por la fijación del ion mineral en óxidos de $\mathrm{Fe}$ y $\mathrm{Al}$, que reducen su disponibilidad para las plantas (Sánchez, 1976; Miller, Schuur, \& Chadwick, 2001). Contrario a ello, el $\mathrm{N}$ del suelo, por ser fijado biológicamente, se considera menos limitante que el $\mathrm{P}$ en suelos tropicales antiguos (Walker \& Syers, 1976; Vitousek, 1984). Asimismo, recientemente se ha reconocido la significativa limitación que ejercen otros macronutrientes, como el K, sobre la PPN (Wright et al., 2011); sin embargo, éstos han sido pobremente evaluados en estos ecosistemas tropicales de baja altitud.

El crecimiento diamétrico arbóreo (CDA) ha sido utilizado para evaluar la limitación de nutrientes en bosques tropicales (Wright et al., 2011; Baribault, Kobe, \& Finley, 2012), debido a que representa el $25 \%$ de la PPN (Malhi, Doughty, \& Galbraith, 2011). En este sentido, se han realizado experimentos de fertilización para evaluar el efecto de la aplicación de nutrientes sobre el CDA en bosques lluviosos tropicales maduros (Mirmanto, Proctor, Green, Nagy, \& Suriantata, 1999; Newbery et al., 2002; Wright et al., 2011; Sullivan et al., 2014; Sayer \& Banin, 2016, entre otros). Sin embargo, tales experimentos han mostrado patrones y tendencias contradictorias. Por ejemplo, Mirmanto et al. (1999) no evidenciaron una respuesta significativa del CDA con la fertilización, luego de adicionar N, P y NP durante un año, en bosques lluviosos tropicales de Dipterocarpaceae con suelos infértiles. Tan solo en algunas especies de rápido crecimiento (Shorea spp.) se observó un mayor CDA con la fertilización, pero tales incrementos no fueron significativos (Mirmanto et al., 1999). De manera similar, Newbery et al. (2002) reportaron que no hubo un efecto significativo sobre el CDA con la aplicación de $\mathrm{P}$ en el suelo en bosques húmedos tropicales de Camerún, en África central.

Contrario a lo anterior, Fisher et al. (2013), después de cuatro años de fertilización, demostraron un incremento en el CDA con la aplicación de $\mathrm{N}+\mathrm{P}$ en bosques tropicales de baja altitud, mientras que Wright et al. (2011) luego de fertilizar durante 11 años con N, P y K en bosques de Panamá, observaron que el CDA incrementó en árboles pequeños con la adición de N y K. Sin embargo, la aplicación de P redujo el CDA. Por su parte, Álvarez-Clare, Mack y Brooks (2013) observaron que la fertilización con $\mathrm{P}$ aumentó el CDA solamente en los árboles pequeños (5-10 cm DAP). Asimismo, con la aplicación de P incrementó el CDA en la especie Socratea exorrhiza, mientras que Goethalsia meiantha presentó una reducción en su CDA con la aplicación de N, P y NP. Estas evidencias sugieren que las respuestas del CDA a la fertilización en bosques tropicales maduros son complejas, puesto que los efectos pueden ser positivos, nulos, o incluso negativos.

Tal variabilidad en las respuestas da lugar a preguntarse por las condiciones que determinan la magnitud y dirección del efecto de la fertilización sobre el CDA en bosques tropicales maduros. La respuesta es compleja, porque se ha observado que el efecto de la disponibilidad de nutrientes (N, P y K) sobre el CDA depende significativamente de otros factores, como la especie vegetal, la densidad de la madera y el grupo funcional evaluado (Baribault et al., 2012). Además, el CDA está influenciado por factores intrínsecos (especie, tamaño y edad de los árboles) y extrínsecos (clima, disponibilidad de recursos, interacciones con árboles vecinos, asociaciones con hongos y bacterias, entre otros) (Salisbury \& Ross, 1994; Lambers, Chapin III \& Pons, 2012), lo cual hace que el proceso sea más complejo.

Además de que los experimentos de fertilización realizados en los bosques tropicales de tierras bajas han sido pocos, cubren un ámbito muy limitado de características ambientales, pues solamente se ha evaluado en zonas con lluvias inferiores a $5000 \mathrm{~mm}$ anuales; por ello se desconocen los efectos de la fertilización en bosques tropicales con precipitaciones superiores. Investigar tales bosques es relevante porque la pluviosidad ejerce una fuerte influencia sobre la disponibilidad de nutrientes y la PPN (Austin \& Vitousek, 1998; Schuur, 2003; Quinto-Mosquera \& Moreno, 2016). Menos aún, se conoce sobre aspectos más específicos del efecto de la fertilización, entre ellos, cómo varía 
según la especie, densidad de madera y grupo funcional.

Particularmente, la región del Pacífico colombiano posee ecosistemas boscosos de tierras bajas y una de las precipitaciones más altas del mundo ( $\approx 10000 \mathrm{~mm}$ anuales) (http:// www.noaa.gov; Poveda, Rojas, Rudas, \& Rangel, 2004), lo cual representa una oportunidad excepcional para estudiar la limitación de nutrientes del suelo sobre el CDA de bosques tropicales con alta precipitación. Con base en lo anterior, el objetivo de investigación planteado, fue evaluar los efectos que tiene la fertilización del suelo con nutrientes ( $\mathrm{N}, \mathrm{P}$ y K), sobre las tasas de CDA a nivel de especie vegetal, categoría diamétrica, densidad de madera y grupos funcionales en bosques pluviales tropicales del Pacífico colombiano.

\section{MATERIALES Y MÉTODOS}

Área de estudio: El presente estudio se realizó en dos bosques pluviales tropicales ubicados en la región del Pacífico colombiano, uno en la localidad de Pacurita (municipio de Quibdó) y otro en la localidad de Opogodó (municipio de Condoto). Los suelos de ambos sitios son Ultisoles (Quinto-Mosquera \& Moreno, 2016). Particularmente, la localidad de Opogodó se encuentra ubicada a $5^{\circ} 04^{\prime} 79^{\prime \prime} \mathrm{N}$ - 7664'47' W, con precipitación de $8000 \mathrm{~mm}$ anuales, altitud de $70 \mathrm{~m}$ y topografía plana. Los suelos son arenosos, muy ácidos, con baja capacidad de intercambio catiónico efectiva (CICE) y contenidos muy bajos de $\mathrm{P}, \mathrm{Mg}$ y Ca. Las concentraciones de $\mathrm{K}$ son intermedias y los valores de materia orgánica (M.O.) y $\mathrm{N}$ total son altos. Las especies arbóreas dominantes son Wettinia quinaria (O.F. Cook \& Doyle) Burret (Arecaceae), Mabea occidentalis Benth (Euphorbiaceae), Calophyllum aurantiacum P.F. Stevens (Calophyllaceae) y Oenocarpus bataua Mart. (Arecaceae) (Quinto-Mosquera \& Moreno, 2016; Quinto-Mosquera, Moreno, Caicedo \& Pérez, 2016). La localidad de Pacurita se encuentra ubicada a $5^{\circ} 41^{\prime} 55.8^{\prime \prime} \mathrm{N}$ - 76³5'59.4” W, con precipitación de 10000 $\mathrm{mm}$ anuales, altitud de $102-140 \mathrm{~m}$ y topografía quebrada. Los suelos son arcillosos y limosos, fuertemente ácidos, con porcentajes altos de saturación de $\mathrm{Al}$, y muy pobres de $\mathrm{P}, \mathrm{Mg}$ y Ca. Asimismo, la disponibilidad de $\mathrm{K}$ es intermedia, mientras M.O. y $\mathrm{N}$ total son altos. Las especies dominantes son C. aurantiacum P.F. Stevens (Calophyllaceae), O. bataua Mart. (Arecaceae), Protium apiculatum Swart (Burseraceae) y Brosimum utile (Kunth) Oken (Moraceae) (Quinto-Mosquera \& Moreno, 2016; Quinto-Mosquera et al., 2016).

En la localidad de Opogodó el muestreo se realizó en tres parcelas permanentes de una hectárea $(100 \times 100 \mathrm{~m})$, instaladas en un bosque ubicado en predios de la Universidad Tecnológica del Chocó "Diego Luis Córdoba". En la localidad de Pacurita el estudio se realizó en dos parcelas permanentes de las mismas dimensiones, ubicadas en una zona de reserva forestal denominada Estación Biológica Pacurita. En ambas localidades, el muestreo se realizó en bosques primarios bien conservados.

Diseño experimental: Para evaluar el efecto de la fertilización del suelo sobre el CDA, se empleó un diseño en bloques completos aleatorizados (Shieh \& Jan, 2004) con cinco tratamientos de fertilización (N, P, K, NPK y Control) y cinco repeticiones. Para ello, cada parcela permanente se dividió en cinco unidades experimentales de $20 \times 100 \mathrm{~m}$ (0.2 ha), separadas por franjas de terreno de $2 \mathrm{~m}$ para reducir el riesgo de que los nutrientes migraran a otras parcelas por efectos de la escorrentía o topografía del suelo. En cada unidad experimental de 20 x 100 m se aplicó aleatoriamente un tratamiento de fertilización. En Pacurita, debido a la influencia de la topografía, la disposición espacial de las unidades experimentales fue paralela al gradiente de inclinación del terreno, para reducir las pérdidas de nutrientes y la contaminación de las unidades vecinas. Cada unidad experimental de 20 x $100 \mathrm{~m}$ se subdividió en cinco unidades de registro de 20 x $20 \mathrm{~m}$, para facilitar la aplicación del fertilizante de modo más homogéneo y la posterior toma de datos de crecimiento de los árboles. 
Aplicación de tratamientos de fertilización: Los fertilizantes se aplicaron por el método de colocación "al voleo" (Alvarado \& Raigosa, 2012). Las dosis añadidas fueron similares a las aplicadas en experimentos realizados en bosques lluviosos tropicales de baja altitud (Mirmanto et al., 1999; Wright et al., 2011). En las unidades de registro de $20 \times 20 \mathrm{~m}$, se aplicaron cuatro dosis iguales de fertilizantes a lo largo del 2014, en los meses de febrero, mayo, agosto y noviembre, así: en el tratamiento de $\mathrm{N}$ se aplicaron $125 \mathrm{~kg}$ N.ha'.año ${ }^{-1}$ en forma de urea $\left(\left(\mathrm{NH}_{2}\right)_{2} \mathrm{CO}\right)$, equivalente a $2.72 \mathrm{~kg}$ de urea por unidad de registro por aplicación; en el tratamiento de $\mathrm{P}$ se adicionaron $50 \mathrm{~kg}$ P.ha${ }^{1}$.año ${ }^{-1}$ en forma de roca fosfórica $\left(\mathrm{H}_{3} \mathrm{PO}_{4}\right)$, equivalente a $1 \mathrm{~kg}$ de $\mathrm{H}_{3} \mathrm{PO}_{4}$ por unidad de registro por aplicación; en el tratamiento de $\mathrm{K}$ se aplicaron $50 \mathrm{~kg} \mathrm{~K} \mathrm{ha}^{-1}$.año ${ }^{-1}$ en forma de cloruro de potasio $(\mathrm{KCl})$ ), equivalente a $1 \mathrm{~kg}$ de $\mathrm{KCl}$ por unidad de registro por aplicación; en el tratamiento de NPK se aplicaron conjuntamente cada una de las dosis mencionadas en los tratamientos anteriores; y en el Control no se aplicaron fertilizantes.

Medición de diámetros y alturas de los árboles: Durante agosto de 2013, 2014 y 2015, se midió la circunferencia a la altura del pecho en $\mathrm{cm}$ de los árboles con diámetro a la altura del pecho (DAP; medido a $1.30 \mathrm{~m}$ sobre el nivel del suelo) $\geq 10 \mathrm{~cm}$ presentes en las parcelas; posteriormente, los valores de circunferencia se transformaron a DAP. Se registraron los árboles vivos, muertos, la causa de mortalidad y los árboles reclutados (que alcanzaron el $\mathrm{DAP} \geq 10 \mathrm{~cm}$ ).

El perímetro del tronco del árbol donde se midió el diámetro (DAP) en el primer censo, se marcó con pintura asfáltica amarilla, con el objetivo de garantizar que posteriores mediciones se realizaran en la misma zona de la primera medición. Tales mediciones se realizaron en áreas libres de nudos o ramas. Además, se registró la ubicación de cada árbol en la cuadrícula. Todos los árboles medidos fueron marcados con placas de aluminio. También se determinó el hábito de crecimiento en las categorías de árbol, bejuco o liana y palma, se anotaron las características vegetativas y observaciones particulares de cada árbol. La altura se midió al $40 \%$ de los árboles registrados en cada unidad; para ello se empleó un clinómetro Suunto a distancias fijas de 15 y $20 \mathrm{~m}$ de observación; así mismo, la altura de los árboles más pequeños (alturas menores a 10-15 m) se determinó con mira graduada o tubos (Mostacedo \& Fredericksen, 2000).

Identificación taxonómica y clasificación en grupos funcionales: Se identificaron todas las morfoespecies hasta el máximo nivel taxonómico posible (indeterminados-NN, especie, género, familia). Esta identificación se llevó a cabo utilizando las claves especializadas de Gentry (1993) y Mahecha (1997); también por comparación con especímenes del herbario CHOCO de la Universidad Tecnológica del Chocó. Posteriormente, se realizó una clasificación de las especies arbóreas en tres grupos, con base en características funcionales sobresalientes: En primer lugar se hizo la división entre monocotilédoneas (Arecaceae) y dicotiledóneas; luego, este segundo grupo se dividió en especies fijadoras de N (Fabaceae, Caesalpinioideae, Mimosoideae) y no fijadoras; con base en Hedin, Brookshire, Menge y Barron (2009), Baribault et al. (2012) y Lambers et al. (2012).

Estimación y clasificación de la densidad de la madera: Para estimar esta variable, se tomaron los valores publicados en dos bases de datos internacionales de densidad de madera, generadas en bosques de la Amazonia (Baker et al., 2004) y en bosques tropicales de diversas regiones del mundo (Brown, 1997); si alguna especie encontrada en las parcelas no estaba incluida en estas bases de datos, se empleó el promedio del género o de la familia correspondiente; para los árboles indeterminados taxonómicamente, se empleó el promedio de densidad de la parcela. Posteriormente, los árboles se agruparon según su densidad de madera, así: baja $\left(0.26-0.49\right.$ g.cm $\left.{ }^{-3}\right)$, media $\left(0.49-0.733\right.$ g.cm $\left.{ }^{-3}\right)$ y alta $\left(0.73-0.97\right.$ g.cm $\left.{ }^{-3}\right)$. 
Para determinar el CDA se empleó la tasa de crecimiento relativo (TCR) por año, la cual se calculó de la siguiente forma: $\mathrm{TCR}=\log \left(\mathrm{DAP}_{\text {final }} / \mathrm{DAP}_{\text {inicial }}\right) /\left(\left(\mathrm{DC}_{\text {final }}-\mathrm{DC}_{\text {ini- }}\right.\right.$ cial $/ 365$ ); donde $\log$ es el logaritmo natural, DAP es el diámetro a altura de pecho y DC son los días contados entre la primera y la última medición (Wright et al., 2011). Se realizaron análisis de crecimiento para las categorías diamétricas siguientes: categoría I $(5 \cdot 0-10.0 \mathrm{~cm}$ de DAP), categoría II (10.1-30.0 cm de DAP), y categoría III ( $>30.0 \mathrm{~cm}$ de DAP).

Para evaluar el efecto de la fertilización (N, P, K, NPK y Control) del suelo sobre la TCR a nivel de localidades, categorías diamétricas, densidad de madera (baja, media y alta), grupos funcionales de especies (Fabaceae, Arecaceae y otras) y especies, se utilizó la prueba no paramétrica de Kruskal-Wallis (Kw) y la prueba de rangos múltiples de Duncan (Hoshmand, 1998), dado que los datos no cumplieron los supuestos (normalidad y homogeneidad de varianzas) para pruebas paramétricas, evaluados con los métodos estadísticos de Bartlett, Hartley y Kurtosis (Hoshmand, 1998). Para el análisis de TCR se tomaron en cuenta todos los árboles (4 015). Los análisis estadísticos se realizaron con los programas Statgraphics Centurion XV (Statistical Graphics Corp., 2002) y en el entorno de programación R (R Core Team, 2012; www.r-project.org/).

\section{RESULTADOS}

En el conjunto de bosques estudiados, la aplicación de $\mathrm{P}$ y $\mathrm{K}$ al suelo generó una reducción significativa en la TCR arbórea con respecto al control $\left(\mathrm{Kw}_{4 ; 4014}=27.1 ; \mathrm{P}\right.$ $<0.01)$. Idéntica tendencia se presentó tanto en Opogodó $\left(\mathrm{Kw}_{4 ;} 2183=17.97 ; \mathrm{P}<0.001\right)$ como en Pacurita $\left(\mathrm{Kw}_{4 ; 1834}=10.45 ; \mathrm{P}<0.01\right)$ (Cuadro 1). A nivel de categorías diamétricas, en Opogodó se registró una reducción significativa de la TCR con la aplicación de los fertilizantes N, P, K y NPK en árboles pequeños (5-10 cm de DAP) $\left(\mathrm{Kw}_{4 ; 463}=27.34 ; \mathrm{P}<0.01\right)$ (Fig. 1A). En árboles medianos se redujo la TCR con la adición de $\mathrm{P}$ y $\mathrm{K}\left(\mathrm{Kw}_{4 ; 1543}=40.9\right.$; $\mathrm{P}<0.01$ ) (Fig. 1C); mientras que en árboles grandes no hubo un efecto significativo de la fertilización sobre la TCR $\left(\mathrm{Kw}_{4 ; 175}=1.37 ; \mathrm{P}\right.$ $=0.849$ ) (Fig. 1E). En Pacurita, se registró una reducción significativa de la TCR con la aplicación de $\mathrm{P}$ y $\mathrm{K}$ en los árboles pequeños $\left(\mathrm{Kw}_{4}\right.$. ${ }_{631}=54.7 ; \mathrm{P}<0.01$ ) (Fig. 1B). En los árboles medianos se observó que la TCR fue menor con la aplicación de $\mathrm{K}$ y NPK $\left(\mathrm{Kw}_{4 ; 1033}=22.8\right.$; $\mathrm{P}<0.01$ ) (Fig. 1D), mientras que en los árboles grandes no hubo un efecto significativo de la fertilización sobre la TCR $\left(\mathrm{Kw}_{4 ; 168}=7.39 ; \mathrm{P}=\right.$ 0.116) (Fig. 1F).

A nivel de densidad de madera, se registró una reducción significativa de la TCR con la aplicación de P y K en árboles de densidad baja

CUADRO 1

Tasa de crecimiento relativo arbórea $\left(\mathrm{mm} \cdot \mathrm{mm}^{-1} \cdot \mathrm{año}^{-1}\right)$ en cinco tratamientos de fertilización del suelo en dos bosques pluviales del Pacífico, Colombia

TABLE 1

Tree relative growth rate $\left(\mathrm{mm} \cdot \mathrm{mm}^{-1} \cdot \mathrm{yr}^{-1}\right)$ in five soil fertilization treatments in two rainforests of the Pacific, Colombia

\begin{tabular}{lccccccc}
\multicolumn{1}{c}{ Localidad } & Control & Nitrógeno & Fósforo & Potasio & NPK & Prueba & P-valor \\
Opogodó & $0.032 \pm 0.15$ & $0.025 \pm 0.12$ & $0.020 \pm 0.1^{* *}$ & $0.015 \pm 0.05^{* *}$ & $0.029 \pm 0.13$ & 17.97 & 0.0012 \\
Pacurita & $0.027 \pm 0.08$ & $0.022 \pm 0.09$ & $0.017 \pm 0.06^{* *}$ & $0.017 \pm 0.07^{* *}$ & $0.022 \pm 0.07$ & 10.45 & 0.0333 \\
General & $0.029 \pm 0.12$ & $0.024 \pm 0.11$ & $0.018 \pm 0.1^{* *}$ & $0.016 \pm 0.06^{* *}$ & $0.026 \pm 0.11$ & 27.104 & 0.0001 \\
\hline
\end{tabular}

Promedio \pm desviación estándar. Los asteriscos $(* *)$ indican diferencias significativas entre los tratamientos y el control, con la prueba de Kruskal-Wallis y estadístico de Rangos Múltiples de Duncan, a un $95 \%$ de confianza.

Average \pm standard deviation. The asterisks $(* *)$ indicate significant differences between treatments and control, with the Kruskal-Wallis and statistical Duncan Multiple Range, a $95 \%$ confidence level. 


\section{Opogodó}
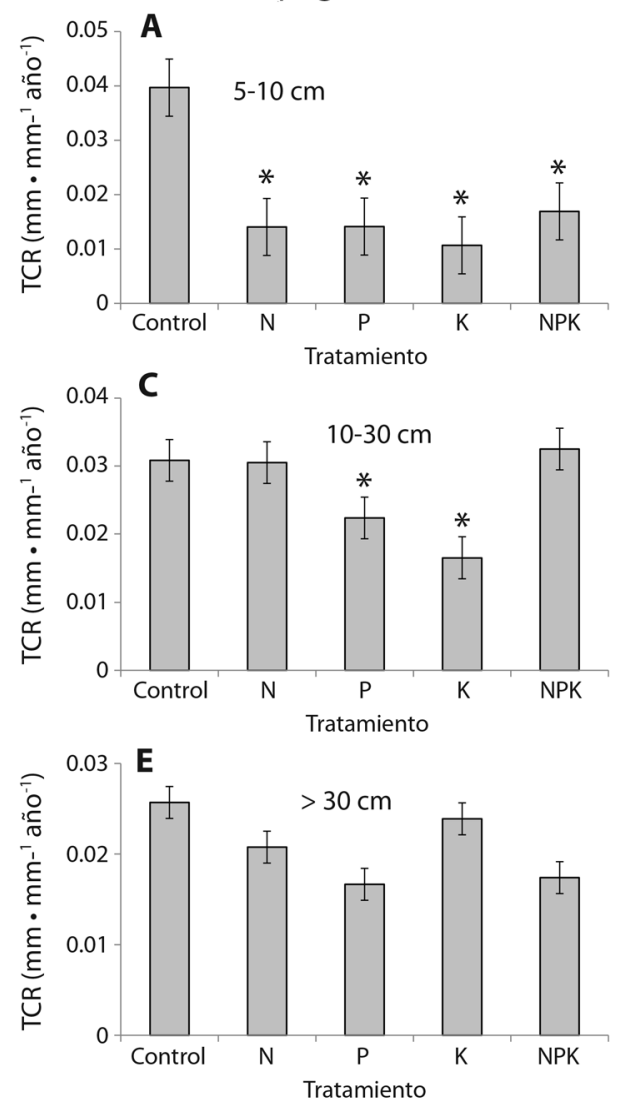

Pacurita
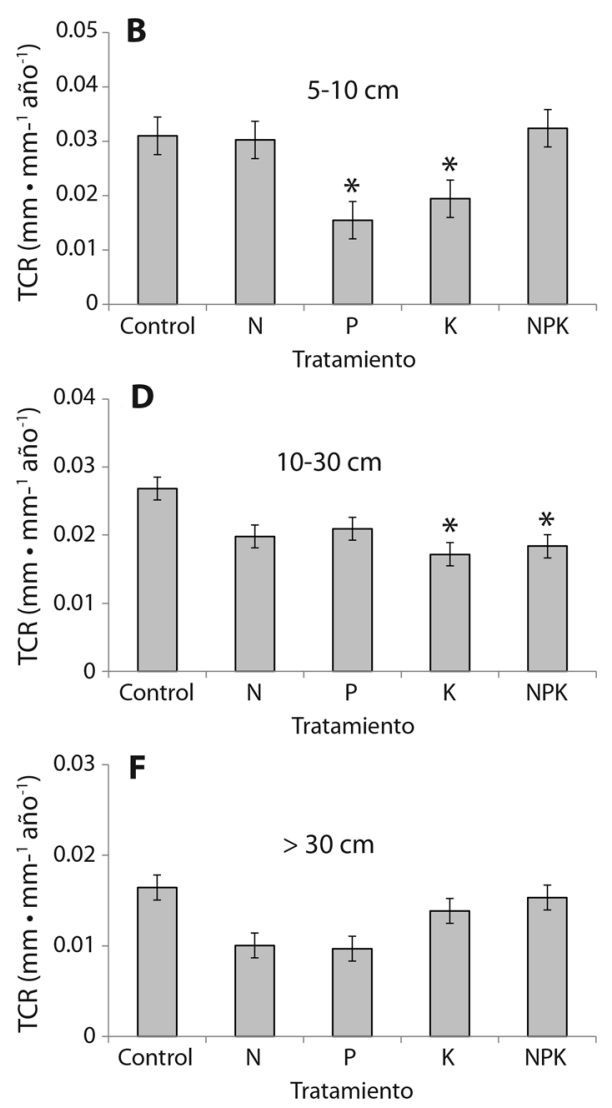

Fig. 1. Efecto de los tratamientos de fertilización sobre la tasa de crecimiento relativo (TCR) en tres categorías diamétricas en bosques pluviales del Pacífico colombiano. Los asteriscos indican diferencias significativas (*) entre los tratamientos y el control.

Fig. 1. Effect of fertilization treatments in the relative growth rate (TCR) in three diameter categories of the Colombian Pacific rainforests. Asterisks $(*)$ mean significant differences between treatments and control.

$\left(\mathrm{Kw}_{4 ; 1038}=17.73 ; \mathrm{P}<0.01\right)$ y alta $\left(\mathrm{Kw}_{4 ; 693}=\right.$ 22.61; P < 0.01) (Fig. 2), mientras que, en los árboles con densidad de madera intermedia, se comprobó menor TCR en los tratamientos con adición de $\mathrm{N}, \mathrm{P}$ y K $\left(\mathrm{Kw}_{4 ; 2283}=86.5 ; \mathrm{P}<0.01\right)$ (Fig. 2). A nivel de los grupos funcionales de especies, se observó que la fertilización no tuvo efecto sobre la TCR de palmas (Arecaceae) $\left(\mathrm{Kw}_{4 ; 394}=9.25 ; \mathrm{P}=0.054\right)$, mientras que en los árboles con capacidad de fijar N simbióticamente, la aplicación de $\mathrm{P}$ y $\mathrm{K}$ produjo menor TCR $\left(\mathrm{Kw}_{4 ; 355}=22.55 ; \mathrm{P}<0.01\right)$ (Fig. 3). En las otras familias dicotiledóneas, con la aplicación de $\mathrm{N}, \mathrm{P}, \mathrm{y} \mathrm{K}$ se registró menor TCR $\left(\mathrm{Kw}_{4}\right.$; ${ }_{3265}=86.26 ; \mathrm{P}<0.01$ ) (Fig. 3).
A nivel de especies arbóreas, se observó que en Inga sp. (Mimosoideae), una de las especies más abundantes (125 árboles) y fijadora de $\mathrm{N}$, la fertilización no causó efectos sobre la TCR $\left(\mathrm{Kw}_{4 ; 125}=8.15 ; \mathrm{P}=0.085\right)$. Sin embargo, las especies Eschweilera sclerophylla (Lecythidaceae) $\left(\mathrm{Kw}_{4 ; 244}=12.69 ; \mathrm{P}=\right.$ $0.012)$ y Miconia sp. (Melastomataceae) $\left(\mathrm{Kw}_{4 \text {; }}\right.$ $\left.{ }_{71}=9.68 ; \mathrm{P}=0.046\right)$ registraron menor $\mathrm{TCR}$ con la aplicación de K (Fig. 4), mientras que Faramea multiflora (Rubiaceae) tuvo menor TCR al recibir fertilización con $\mathrm{N}, \mathrm{P}$ y $\mathrm{K}\left(\mathrm{Kw}_{4}\right.$; ${ }_{70}=11.89 ; \mathrm{P}=0.018$ ) (Fig. 4). En Matisia sp. (Malvaceae) se registró mayor TCR con la aplicación de NPK $\left(\mathrm{F}_{4 ; 66}=2.37 ; \mathrm{P}=0.061\right)$; 

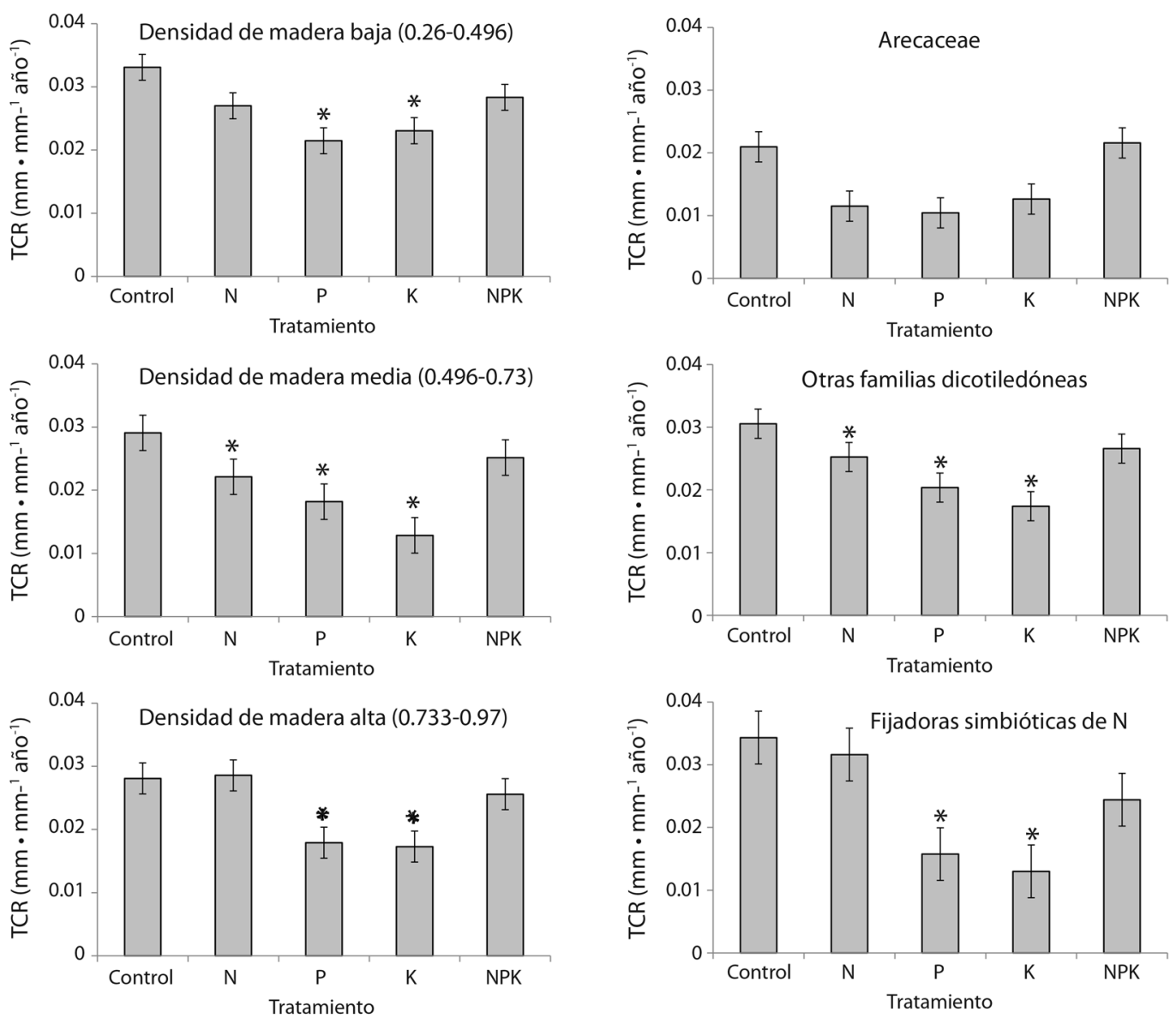

Fig. 2. Efecto de los tratamientos de fertilización sobre la tasa de crecimiento relativo (TCR) en tres niveles de densidad de madera (alta, media y baja). Los asteriscos (*) indican diferencias significativas entre los tratamientos y el control.

Fig. 2. Effect of fertilization treatments on the relative growth rate (TCR) in three levels of wood density (high, medium and low). The asterisks $\left(^{*}\right)$ mean significant differences between treatments and control.

asimismo, en Andira sp. (Fabaceae), se observó mayor TCR con la adición de $\mathrm{N}\left(\mathrm{Kw}_{4 ; 41}=\right.$ 11.01; $\mathrm{P}=0.026)$. En estos últimos dos casos, hubo diferencias con los demás tratamientos de fertilización, pero no con el control (Fig. 4). En el resto de las especies la fertilización no afectó la TCR.

Fig. 3. Efecto de los tratamientos de fertilización sobre la tasa de crecimiento relativo (TCR) de tres grupos funcionales de especies (Arecaceae, especies con fijación simbiótica de N (Fabaceae, Caesalpinioideae, Mimosoideae) y otras dicotiledóneas).

Fig. 3. Effect of fertilization treatments on the relative growth rate (TCR) of three functional groups of species (Arecaceae, symbiotic N-fixing species (Fabaceae, Caesalpinioideae, Mimosoideae), and other dicotyledonous).

\section{DISCUSIÓN}

Las reducciones de la TCR con la aplicación de nutrientes, registradas en este estudio, también se han documentado en otros bosques lluviosos tropicales de baja altitud. Por ejemplo, Wright et al. (2011) observaron 

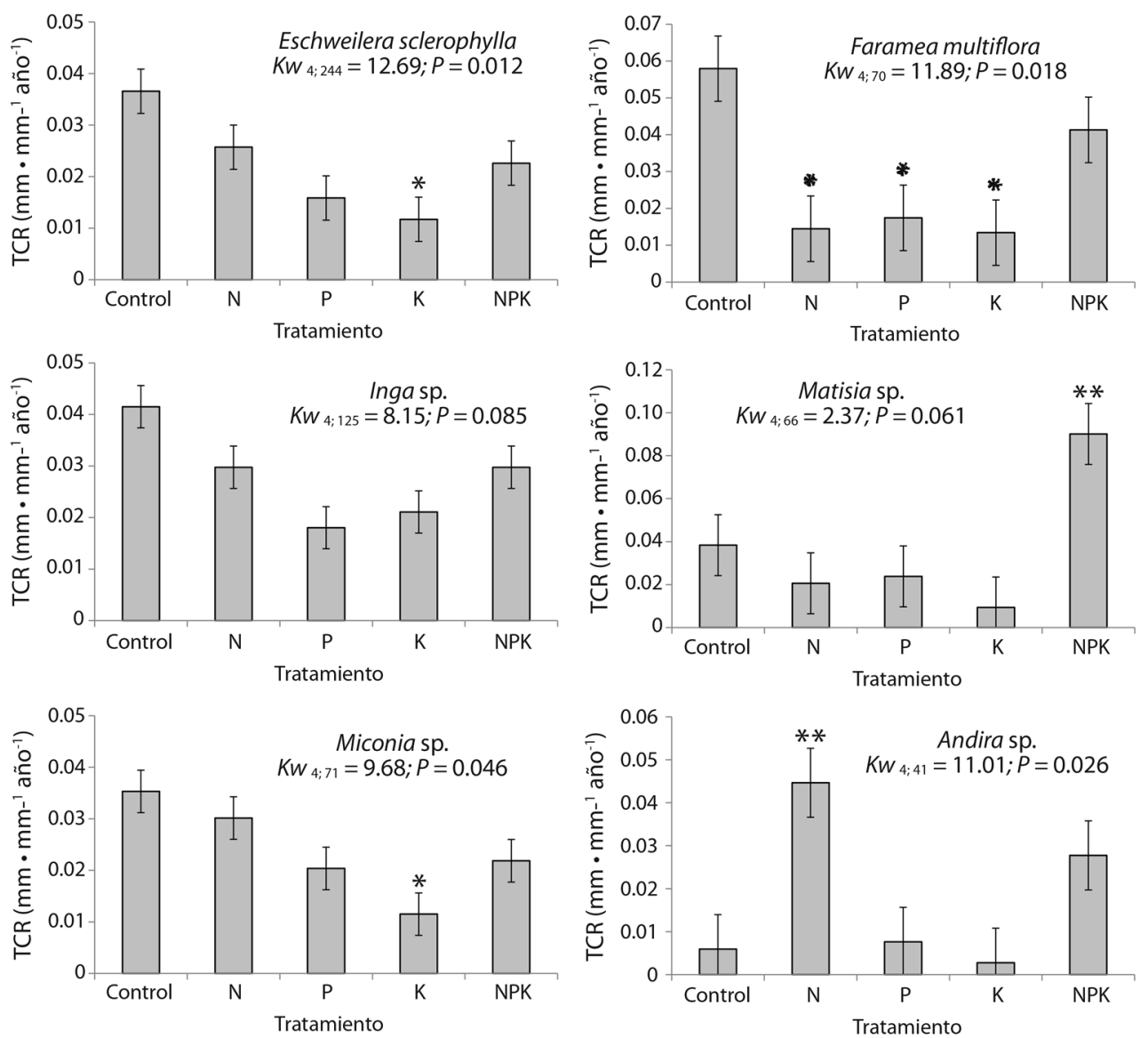

Fig. 4. Efecto de los tratamientos de fertilización sobre la tasa de crecimiento diamétrico (TCR) de seis especies comunes. Los asteriscos $\left(^{*}\right)$ indican diferencias significativas entre TCR de los tratamientos y el control. Los dos asteriscos (**) indican diferencias significativas en TCR entre los tratamientos, pero no con el control.

Fig. 4. Effect of fertilization treatments on diameter growth rate (TCR) of six common species. Asterisks $\left({ }^{*}\right)$ indicate significant differences in TCR between treatments and control. The two asterisks $(* *)$ indicate significant differences in TCR between treatments, but not with the control.

que la aplicación de P redujo la TCR en árboles mayores de $10 \mathrm{~cm}$ de DAP, aunque la adición de $\mathrm{N}$ y $\mathrm{K}$ aumentó el crecimiento en árboles pequeños. Cusack, Silver, Torn y McDowell (2011) observaron una menor tasa de CDA en árboles pequeños $(<15 \mathrm{~cm}$ de DAP) y medianos (15-20 cm de DAP) con la aplicación de N en bosques de Costa Rica. Por su parte, Álvarez-Clare et al. (2013) señalan que la aplicación de N, P y NP disminuyó la TCR en árboles de Goethalsia meiantha (Malvaceae), luego de tres años de fertilización.
A la vez que el CDA disminuyó con la fertilización del suelo en los bosques pluviales del Pacífico colombiano, se registró un incremento en la PPN de raíces finas y de hojarasca del bosque, especialmente en la localidad de Opogodó (Quinto-Mosquera, 2016). Estos resultados sugieren que la aplicación de nutrientes (principalmente $\mathrm{P}$ y $\mathrm{K}$ ) generó un cambio en los patrones de asignación de carbono; es decir, que hubo una redistribución de la PPN aérea y subterránea del bosque, tal vez como respuesta al déficit de estos nutrientes en el ecosistema. 
Asimismo, la aplicación de cada nutriente $(\mathrm{N}$, P, K y NPK) afectó de forma diferente los distintos componentes de la PPN del ecosistema; resultados similares se registran en los bosques de Panamá, donde la aplicación factorial de N, $\mathrm{P}$ y K afectó diferencialmente los componentes de la PPN (Kaspari et al., 2008; Wright et al., 2011; Wurzburger \& Wright, 2015).

La disminución del CDA y el incremento de la PPN de raíces finas y hojarasca con la fertilización, sugiere que los nutrientes adicionados fueron empleados para producir estructuras destinadas a realizar fotosíntesis (renovación de hojas) y a la adquisición de nutrientes y agua del suelo (raíces finas), y no tanto al crecimiento diamétrico. Esta hipótesis concuerda con lo planteado por Bloom, Chapin III y Mooney (1985), quienes concluyeron que las plantas responden homeostáticamente al desequilibrio de recursos en el ambiente (por ejemplo, déficit de nutrientes), mediante la asignación de nueva biomasa (PPN) para la adquisición de los recursos que son más limitantes del crecimiento (Bloom et al., 1985).

Lo anterior a su vez refleja el alto costo energético de la adquisición de recursos y del CDA en condiciones oligotróficas (Bloom et al., 1985). En concordancia con ello, algunos estudios realizados en bosques tropicales han evidenciado incrementos en el contenido de nutrientes en hojas y raíces finas con la fertilización (Kaspari et al., 2008; Ostertag, 2010; Cusack et al., 2011; Mayor, Wright, \& Turner, 2014; Álvarez-Clare \& Mack, 2015; Mo et al., 2015). Asimismo, se han detectado correlaciones significativas entre la concentración foliar de nutrientes y la PPN (Vitousek, 1984; Cleveland et al., 2011; Mercado et al., 2011; Álvarez-Clare \& Mack, 2015), lo cual sugiere que con la fertilización, no solo se incrementa la PPN del bosque, sino también el contenido y tasa de reciclaje de nutrientes en los tejidos vegetales (Kaspari et al., 2008).

Los experimentos de Wright et al. (2011) y Álvarez-Clare et al. (2013) mostraron que los árboles pequeños son más sensibles que los grandes a la adición de nutrientes en bosques tropicales. Este patrón también se evidenció en el presente estudio, aunque a diferencia de los experimentos mencionados, el CDA de los árboles pequeños y medianos respondió negativamente a la aplicación de nutrientes. El hecho de que los árboles pequeños hayan sido más sensibles a la fertilización se debe probablemente a que éstos se suelen encontrar bajo condiciones de luminosidad limitada en el sotobosque, además de que presentan un sistema radical más superficial y menos desarrollado, por lo que son más vulnerables a los incrementos extras de nutrientes en el sistema (Wright et al., 2011; Sayer \& Banin, 2016). Asimismo, los árboles medianos y grandes tienden a inmovilizar más los nutrientes aplicados en la fertilización, por lo que es más difícil detectar sus efectos (Miller, 1981; Santiago, 2015).

Se ha planteado la hipótesis de que las características ecofisiológicas y requerimientos nutricionales de las especies están asociados con la densidad de madera. Se considera que especies con madera de baja densidad presentan altas tasas de CDA y son más sensibles a cambios en la disponibilidad de nutrientes del suelo, mientras que las especies con madera de alta densidad son más tolerantes a la sombra, crecen lentamente y responden menos a cambios en los nutrientes del suelo (Chave et al. 2009; Poorter et al., 2010; Baribault et al. 2012). Por tal razón, en el presente estudio se esperaba que la fertilización tuviera efectos diferenciales sobre el CDA en función de la densidad de la madera de las especies. Sin embargo, los resultados no apoyaron tal predicción, puesto que las respuestas del CDA a la fertilización fueron similares entre los diferentes grupos de densidad de madera. Al parecer, con la adición de nutrientes durante los primeros años, la estrategia de promover la producción de tejido fotosintético (hojas) y radicular para la captura de recursos, es común en todos los tipos de densidad de madera del bosque. Posiblemente, si continúa la fertilización durante varios años, los efectos diferenciales se evidenciarían con mayor proporción en los tejidos leñosos y en el CDA. Seguramente las especies con menor densidad de madera tendrán mayores respuestas. 
A nivel de grupos funcionales de especies, se observaron diferencias en las respuestas del CDA a la fertilización. A pesar de que la respuesta del CDA a la aplicación de $\mathrm{P}$ y $\mathrm{K}$ de las familias fijadoras de $\mathrm{N}$ fue similar a la de las otras dicotiledóneas y se caracterizó por su disminución, las respuestas a la aplicación de $\mathrm{N}$ fueron diferentes: mientras que en las leguminosas no hubo respuesta, en el grupo de otras dicotiledóneas tal aplicación disminuyó significativamente el CDA. En efecto, las leguminosas por su capacidad de fijación simbiótica de $\mathrm{N}$ atmosférico (Hedin et al., 2009), pueden utilizar tal $\mathrm{N}$ para suplir sus necesidades; por tanto, la sensibilidad a la aplicación de $\mathrm{N}$ debería ser menor, lo cual efectivamente ocurrió.

La disminución del CDA en el grupo de otras dicotiledóneas al aplicar independientemente $\mathrm{N}, \mathrm{P}$ y K (y en las leguminosas a la aplicación de $\mathrm{P}$ y K), sigue el mismo patrón descrito atrás para el CDA por categoría diamétrica y de densidad. Por tanto, se puede explicar de la misma forma: cuando un nutriente es escaso, la respuesta de corto plazo después de aplicarlo es aumentar la producción de material lábil (hojas y raíces) en detrimento del crecimiento leñoso.

Finalmente, en este estudio se encontró que las respuestas del CDA de las especies arbóreas frente a la fertilización no fueron homogéneas. Algunas respondieron positivamente (Matisia sp. con NPK, y Andira sp. con $\mathrm{N}$ y NPK), otras negativamente (Eschweilera sclerophylla, Miconia sp., Inga sp., y Faramea multiflora), pero la mayoría no respondieron a la aplicación de nutrientes, lo cual también se evidenció en los experimentos de Mirmanto et al. (1999) y Álvarez-Clare et al. (2013). Para encontrar respuestas a tal variabilidad, es útil la revisión reciente de experimentos de fertilización en el trópico por Santiago (2015), quien concluyó que las mayores respuestas de los árboles a la aplicación de nutrientes se pueden agrupar en dos: por un lado, las respuestas a corto plazo, que incluyen aumento de concentración foliar de nutrientes, tasa fotosintética, conductancia estomática y eficiencia en el uso del agua. Por otro lado, las respuestas a largo plazo que incluyen incremento en el CDA, cambios en la biomasa de raíces y colonización por micorrizas, reducción en la capacidad de captura de nutrientes, entre otros (Santiago, 2015). En concordancia con esa revisión, el presente estudio no evidencia aumento de CDA a corto plazo, después de la fertilización. No obstante, la variación en las respuestas entre especies amerita un análisis más detallado, que requiere de información autoecológica relevante, no disponible todavía, por lo cual tal análisis escapa a los objetivos de este estudio.

\section{AGRADECIMIENTOS}

Esta investigación fue financiada por el Convenio 0010-2013 firmado entre la Universidad Tecnológica del Chocó "Diego Luis Córdoba", la Universidad Nacional de Colombia Sede Medellín y el Departamento Administrativo de Ciencia, Tecnología e Innovación - COLCIENCIAS, en el marco del proyecto titulado: "Evaluación del efecto de la fertilización del suelo sobre la productividad primaria neta de bosques pluviales tropicales del departamento del Chocó (CÓDIGO: 1128-56935113)". Agradecemos la hospitalidad y colaboración de los habitantes de las localidades de Opogodó (municipio de Condoto) y Pacurita (municipio de Quibdó).

\section{RESUMEN}

El crecimiento diamétrico arbóreo (CDA) es un componente fundamental de la productividad primaria neta (PPN) y del almacenamiento de carbono en ecosistemas boscosos, por lo cual cumple un papel importante en la mitigación del cambio climático global. Se ha planteado la hipótesis de que en los bosques lluviosos tropicales de baja altitud el CDA está limitado por la disponibilidad edáfica de nutrientes; sin embargo, pocas evidencias experimentales la apoyan, especialmente en bosques con alta precipitación ( $>5000 \mathrm{~mm}$ anuales). Este estudio evalúa los efectos de la fertilización del suelo sobre el CDA en bosques pluviales tropicales del Pacífico Colombiano, una de las zonas más lluviosas del mundo. Tales efectos se evaluaron a nivel de localidades, categorías diamétricas, densidad de madera y grupos funcionales. Para ello, se realizaron dos inventarios arbóreos (2014 y 2015), se aplicaron cinco tratamientos de fertilización (nitrógeno-N, fósforo-P, 
potasio-K, NPK y Control) y se determinó el CDA en cinco parcelas permanentes de una hectárea. Se encontró que la aplicación de N y NPK tuvo poco efecto sobre el CDA con respecto al control, mientras que la adición de $\mathrm{P}$ y $\mathrm{K}$ produjo reducción significativa en la tasa de crecimiento relativo arbórea con respecto al control a nivel de las localidades, en árboles pequeños y medianos, en todas las densidades de madera (baja, media y alta) y en el grupo de plantas dicotiledóneas. Aunque los resultados no corroboraron la hipótesis de limitación de nutrientes sobre el CDA en los bosques estudiados, se evidenció que durante los primeros años de fertilización (principalmente con P y K) ocurrieron cambios en los patrones de PPN aérea y subterránea, probablemente para maximizar la fotosíntesis y la adquisición de nutrientes del suelo.

Palabras clave: balance del carbono, Chocó biogeográfico, nitrógeno, fósforo, potasio, tasa de crecimiento relativo, productividad primaria neta.

\section{REFERENCIAS}

Alvarado, A., \& Raigosa, J. (2012). Nutrición y Fertilización Forestal en Regiones Tropicales. San José, Costa Rica: Asociación Costarricense de la Ciencia del Suelo.

Álvarez-Clare, S. \& Mack, M. C. (2015). Do foliar, litter, and root nitrogen and phosphorus concentrations reflect nutrient limitation in a lowland tropical wet forest? PLoS ONE, 10(4), e0123796. doi 10.1371/ journal.pone. 0123796

Álvarez-Clare, S., Mack, M. C., \& Brooks, M. (2013). A direct test of nitrogen and phosphorus limitation to net primary productivity in a lowland tropical wet forest. Ecology, 94(7), 1540-1551.

Austin, A. T., \& Vitousek, P. M. (1998). Nutrient dynamics on a precipitation gradient in Hawai'i. Oecologia, 113(4), 519-529.

Baker, T. R., Phillips, O. L., Malhi, Y., Almeida, S., Arroyo, L., Di Fiore, A., Erwin, T., (...), \& Vasquez Martinez, R. (2004). Variation in wood density determines spatial patterns in Amazonian forest biomass. Global Change Biology, 10(5), 545-562.

Baribault, T. W., Kobe, R. K., \& Finley, A. O. (2012). Tropical tree growth is correlated with soil phosphorus, potassium, and calcium, though not for legumes. Ecological Monographs, 82(2), 189-203.

Bloom, A. J., Chapin III, F. S., \& Mooney, H. A. (1985). Resource limitation in plants - an economic analogy. Annual Review of Ecology and Systematics, $16,363-392$.

Brown, S. (1997). Estimating biomass and biomass change of tropical forests: A primer. Food and Agriculture Organization, Roma. (UN FAO Forestry Paper; no. 134). Recuperado de http://www.fao.org/docrep/ w4095e/w4095e00.HTM

Chave, J., Coomes, D., Jansen, S., Lewis, S. L., Swenson, N. G., \& Zanne, A. E. (2009). Towards a worldwide wood economics spectrum. Ecology Letters, 12(4), 351-366.

Cleveland, C. C., Townsend, A. R. Taylor, P., AlvarezClare, S., Bustamante, M., Chuyong, G., Dobrowski, S. Z., (...), \& Wieder, W. R. (2011). Relationships among net primary productivity, nutrients and climate in tropical rain forest: a pan-tropical analysis. Ecology Letter, 14(9), 939-947.

Cusack, D. F., Silver, W. L., Torn, M. S., \& McDowell, W. H. (2011). Effects of nitrogen additions on above and belowground carbon dynamics in two tropical forests. Biogeochemistry, 104(1), 203-225.

Dalling, J. W., Heineman, K., Lopez, O. R., Wright, S. J., \& Turner, B. L. (2016). Nutrient availability in tropical rain forests: The paradigm of Phosphorus limitation. In G. Goldstein, \& L.S. Santiago (Eds.), Tropical tree physiology. Adaptations and responses in a changing environment (pp. 261-273). Switzerland: Springer International Publishing.

Dixon, R. K., Brown, S., Houghton, R. A., Solomon, A. M., Trexler, M. C., \& Wisniewski, J. (1994). Carbon pools and flux of global forest ecosystems. Science, 263(5144), 185-190.

Fisher, J. B., Malhi, Y., Torres, I. C., Metcalfe, D. B., van de Weg, M. J., Meir, P., Silva-Espejo, J. E., \& Huasco, W. H. (2013). Nutrient limitation in rainforests and cloud forests along a 3,000-m elevation gradient in the Peruvian Andes. Oecologia, 172(3), 889-902.

Gentry, A. (1993). A field guide to the families and genera of woody plants of Northwest South American (Colombia, Ecuador, Peru). Washington, DC, USA: Conservation International.

Hedin, L. O., Brookshire, J., \& Menge, D., \& Barron, A. R. (2009). The Nitrogen Paradox in Tropical Forest Ecosystems. The Annual Review of Ecology, Evolution, and Systematics, 40, 613-635.

Hoshmand, A. R. (1998). Statistical Methods for Environmental y Agricultural Sciences. Second edition. New York, USA: CRC Press LLC.

Panel Intergubernamental de Cambio Climático (IPCC). (2014). Cambio climático 2014: Informe de síntesis. In R. K. Pachauri, \& L. A. Meyer (Eds.), Contribución de los Grupos de trabajo I, II y III al Quinto Informe de Evaluación del Grupo Intergubernamental de Expertos sobre el Cambio Climático. Ginebra, Suiza: IPCC. Recuperado de http://www.ipcc.ch/ index.htm

Kaspari, M., Garcia, M. N., Harms, K. E., Santana, M. Wright, S. J., \& Yavitt, J. B. (2008). Multiple 
nutrients limit litterfall and decomposition in a tropical forest. Ecology Letters, 11(1), 35-43.

Lambers, H., Chapin III, F. S., \& Pons, T. L. (2012). Plant Physiological Ecology (Second Ed.). New York, USA: Springer Science Business Media, LLC.

Mahecha, G. (1997). Fundamentos y metodologías para la identificación de plantas (Proyecto Biopacifico, Ministerio del Medio Ambiente, GEF-PNUD). Santa Fé de Bogotá, Colombia: Lerner Ltda.

Malhi, Y., Doughty, C., \& Galbraith, D. (2011). The allocation of ecosystem net primary productivity in tropical forests. Philosophical Transactions of the Royal Society B: Biological Sciences, 366(1582), 3225-3245.

Mayor, J. R., Wright, S. J., \& Turner, B. L. (2014). Speciesspecific responses of foliar nutrients to long term nitrogen and phosphorus additions in a lowland tropical forest. Journal of Ecology, 102(1), 36-44.

Mercado, L. M., Patiño, S., Domingues, T. F., Fyllas, N. M., Weedon, G. P., Sitch, S., (...), \& Lloyd, J. (2011). Variations in Amazon forest productivity correlated with foliar nutrients and modelled rates of photosynthetic carbon supply. Philosophical Transactions of the Royal Society B, 366(1582), 3316-3329.

Miller, H. G. (1981). Forest fertilization: some guiding concepts. Forestry, 54(2), 157-168.

Miller, A. J., Schuur, E. A. G., \& Chadwick, O. A. (2001). Redox control of phosphorus pools in Hawaiian montane forest soils. Geoderma, 102(3), 219-237.

Mirmanto, E., Proctor, J., Green, J., Nagy, L., \& Suriantata. (1999). Effects of nitrogen and phosphorus fertilization in a lowland evergreen rainforest. Philosophical Transactions of the Royal Society B, 354 (1391), 1825-1829.

Mo, Q., Zou, B., Li, Y., Chen, Y., Zhang, W., Mao, R., Ding, Y., (...), \& Wang, F. (2015). Response of plant nutrient stoichiometry to fertilization varied with plant tissues in a tropical forest. Scientific Reports, 5(14605), 1-12.

Mostacedo, B., \& Fredericksen, T. (2000). Manual de Métodos Básicos de Muestreo y Análisis en Ecología Vegetal. Santa Cruz, Bolivia: Editora El País.

Newbery, D. M., Chuyong, G. B., Green, J. J., Songwe, N. C., Tchuenteu, F., \& Zimmermann, L. (2002). Does low phosphorus supply limit seedling establishment and tree growth in groves of ectomycorrhizal trees in a central African rainforest? New Phytologist, $156(2), 297-311$

Ostertag, R. (2010). Foliar nitrogen and phosphorus accumulation responses after fertilization: an example from nutrient-limited Hawaiian forests. Plant and Soil, 334(1), 85-98.
Paoli, G. D., \& Curran, L. M. (2007). Soil nutrients limit fine litter production and tree growth in mature lowland forest of southwestern Borneo. Ecosystems, 10(3), 503-518.

Phillips, O. L., Malhi, Y., Higuchi, N., Laurance, W., Núñez, P., Vásquez, M., Laurance, S., Ferreira, L. Stern, M., Brown, S., \& Grace, J. (1998). Changes in the carbon balance of tropical forest: Evidence from long-term plots. Science, 282(5388), 439-442.

Poorter, L., McDonald, I., Alarcon, A., Fichtler, E., Licona, J. C., Pena-Claros, M., (...), \& Sass-Klaassen, U. (2010). The importance of wood traits and hydraulic conductance for the performance and life history strategies of 42 rainforest tree species. New Phytologist, 185(2), 481-492.

Poveda, I. C., Rojas, C., Rudas, A., \& Rangel, O. (2004). El Chocó biogeográfico: Ambiente Físico. In O. Rangel (Ed.), Colombia Diversidad Biótica IV. El Chocó biogeográfico/ Costa Pacifica. Bogotá, Colombia: Instituto de Ciencias Naturales. Universidad Nacional de Colombia.

Quinto-Mosquera, H., Moreno, F. H., Caicedo, M. H., \& Pérez, L. M. (2016). Biomasa de raíces finas y fertilidad del suelo en bosques pluviales tropicales del Pacífico Colombiano. Colombia Forestal, 19(1), 53-66.

Quinto-Mosquera, H., \& Moreno, F. H. (2016). Precipitation effects on soil characteristics in tropical rain forests of the Chocó biogeographical region. Revista Facultad Nacional de Agronomía Medellín, 69(1), 7813-7823.

Quinto-Mosquera, H. (2016). Evaluación del efecto de la fertilización del suelo sobre la productividad primaria neta de bosques pluviales tropicales del departamento del Chocó (Tesis de doctorado). Universidad Nacional de Colombia sede Medellín, Medellín, Colombia.

Salisbury, F. B. \& Ross, C. W. (1994). Fisiología Vegetal (4ta. Ed.). México D.F: Grupo Editorial Iberoamérica S.A.

Sánchez, P. A. (1976). Properties and management of soils in the tropics. New York, USA: WileyInterscience Publications.

Santiago, L. S. (2015). Nutrient limitation of ecophysiological processes in tropical trees. Trees, 29(5), 1291-1300.

Sayer, E. J., \& Banin, L. F. (2016). Tree nutrient status and nutrient cycling in tropical forest-Lessons from fertilization experiments. In G. Goldstein, \& L.S. Santiago (Eds.), Tropical tree physiology. Adaptations and responses in a changing environment (pp. 275-297). Switzerland: Springer International Publishing. 
Schuur, E. A. G. (2003). Net primary productivity and global climate revisited: the sensitivity of tropical forest growth to precipitation. Ecology, 84(5), 1165-1170.

Shieh, G., \& Jan, S. (2004). The effectiveness of randomized complete block design. Statistica Neerlandica, 58(1), 111-124.

Statistical Graphics Corp. (2002). Statgraphics Plus Centurium XV. Version 5.1. Recuperado de http://www. statgraphics.com/

Sullivan, B. W., Alvarez-Clare, S., Castle, S. C., Porder, S., Reed, S. C., Schreeg, L., Cleveland, C. C., \& Townsend, A. R. (2014). Assessing nutrient limitation in complex forested ecosystems: alternatives to large-scale fertilization experiments. Ecology, 95(3), 668-681.

R Core Team. (2012). $R$ : A language and environment for statistical computing. Vienna, Austria: R Foundation for Statistical Computing. Recuperado de http:// www.R-project.org/
Vitousek, P. M., \& Farrington, H. (1997). Nutrient limitation and soil development: Experimental test of a biogeochemical theory. Biogeochemistry, 37(1), 63-75.

Vitousek, P. M. (1984). Litterfall, nutrient cycling and nutrient limitation in tropical forests. Ecology, 65(1), 285-298.

Walker, T. W. \& Syers. J. K. (1976). The fate of phosphorus during pedogenesis. Geoderma, 15(1), 1-19.

Wright, S. J., Yavitt, J. B., Wurzburger, N., Turner, B. L., Tanner, E. V. J., Sayer, E. J., Santiago, L. S., (...), \& Corre, M. D. (2011). Potassium, phosphorus, or nitrogen limit root allocation, tree growth, or litter production in a lowland tropical forest. Ecology, 92(8), 1616-1625.

Wurzburger, N. \& Wright, S. J. (2015). Fine-root responses to fertilization reveal multiple nutrient limitation in a lowland tropical forest. Ecology, 96(8), 2137-2146. 